\title{
ON MULTIPLIERS OF SEGAL ALGEBRAS
}

\author{
M. DUTTA AND U. B. TEWARI
}

\begin{abstract}
Let $T$ be a multiplier of a Segal algebra $S$ on a locally compact abelian group $G$. We prove that $T^{2}(S)$ is closed if and only if $T$ is a product of an idempotent and an invertible multiplier. We also show that the techniques developed in the proof of this theorem can be used to obtain some other known results.
\end{abstract}

1. Introduction. Let $S$ be a Segal algebra on a locally compact abelian group $G$ with dual group $\Gamma$. (For definition and examples of Segal algebras, see [4].) A bounded linear operator $T$ on $S$ is called a multiplier if for all $f$, $g \in S, T(f * g)=f *(T g)=(T f) * g$. If $T$ is a multiplier of $S$ then there exists a bounded continuous function $\hat{T}$ on $\Gamma$ such that for all $f \in S$ and for all $\gamma \in \Gamma \widehat{T f}(\gamma)=\hat{T}(\gamma) \hat{f}(\gamma)$ and $\|\hat{T}\|_{\infty} \leqslant\|T\|$. The set of multipliers of $S$, denoted by $M(S)$, forms a commutative Banach algebra of operators under the operator norm (for a detailed discussion of multipliers, see [2]).

In $\$ 2$, we prove the following theorem.

THEOREM 1. $T^{2}(S)$ is closed if and only if $T$ is a product of an idempotent and an invertible multiplier.

In [1], the special case of this theorem for $S=L^{1}(G)$ is proved. The proof immediately generalizes to all Banach algebras satisfying the special hypothesis mentioned in $\$ 4$ of [1]. Our result, obtained by similar methods, is stronger as there are Segal algebras which do not satisfy this hypothesis. For example, we can take $G=T$, the circle group, and take

$S=\left\{f \in L^{1}(T): \sum_{n=-\infty}^{\infty}|n \hat{f}(n)|<\infty\right.$ with $\left.\|f\|_{s}=\|f\|_{L^{1}(T)}+\sum_{n=-\infty}^{\infty}|n \hat{f}(n)|\right\}$.

In $\$ 3$, we apply the results of $\$ 2$ to prove the following theorems.

THEOREM 2. If $T$ is an isometric multiplier on $S$ then $T$ is surjective and for all $\gamma \in \Gamma,|\hat{T}(\gamma)|=1$.

THEOREM 3. If $G$ is noncompact then the only compact multiplier of $S$ is the zero operator.

Both of these results have been earlier proved by other methods (see [3]).

2. Before proving Theorem 1, we shall prove a few lemmas.

Received by the editors September 29, 1977.

AMS (MOS) subject classifications (1970). Primary 43A22.

Key words and phrases. Locally compact abelian groups, Segal algebra, multiplier.

○ American Mathematical Society 1978 
LEMMA 1. If $I$ is an ideal in $S$ and $T(I)$ is closed for some $T \in M(S)$ then $\hat{T}$ is bounded away from zero on $W=\Gamma \backslash$ hull $T(I)=\Gamma \backslash\left(\hat{T}^{-1}(0) \cup\right.$ hull $\left.I\right)$.

Proof. Since $T$ is continuous, we can take $I$ to be closed without loss of generality. Now, by the open mapping theorem there exists $K>0$ such that for any $f \in T(I)$ we can find $g \in I$ such that $f=T g$ and $\|g\|_{s} \leqslant K\|f\|_{s}$.

Let $\gamma \in W$. Consider $\gamma$ as an element of $S^{*}$, the dual of $S$ and let $\|\gamma\|=K_{1}>0$. Then there exists $f_{1} \in S$ such that $\hat{f}_{1}(\gamma)=1$ and $\left\|f_{1}\right\|_{s} \leqslant$ $2 / K_{1}$. Choose $V \subset W$ such that $V$ is a compact neighbourhood of $\gamma$. (Note that $W$ is open.) Choose $f_{2} \in L^{1}(G)$ such that $\hat{f}_{2}(\gamma)=1,\left\|f_{2}\right\|_{L^{\prime}(G)}=1$ and $\hat{f}_{2}$ is supported in $V$. This is possible by 2.6.1 of [6]. For $f=f_{2} * f_{1}$, we have $f \in S,\|f\|_{s} \leqslant\left\|f_{2}\right\|_{L^{\prime}(G)}\left\|f_{1}\right\|_{s} \leqslant 2 / K_{1}, \hat{f}(\gamma)=1$ and $\hat{f}$ is supported in $V$.

Since $T(I)$ is a closed ideal (T(I) is an ideal since $T$ is a multiplier) and $\hat{f}$ has compact support disjoint from hull $T(I)$, we get $f \in T(I)$. Hence we can find $g \in I$, such that $f=T g$ and $\|g\|_{s} \leqslant K\|f\|_{s} \leqslant 2 K / K_{1}$. Therefore,

$$
\begin{aligned}
1 & =\hat{f}(\gamma)=\hat{T}(\gamma) \hat{g}(\gamma)=|\hat{T}(\gamma)||\hat{g}(\gamma)| \\
& \leqslant|\hat{T}(\gamma)|\|\gamma\|\|g\|_{s} \leqslant|\hat{T}(\gamma)| 2 K .
\end{aligned}
$$

Therefore, $|\hat{T}(\gamma)| \geqslant 1 / 2 K$.

Since $\gamma$ is an arbitrary element of $W$ we conclude that $\hat{T}$ is bounded away from zero on $W$. This completes the proof.

LemMa 2. Let $T, T_{1}$ be in $M(S)$ such that $\hat{T}_{1}(\gamma)=(\hat{T}(\gamma))^{-1}$ for all $\gamma \in \Gamma$ satisfying $\hat{T}(\gamma) \neq 0$. Then $T$ is a product of an idempotent and an invertible multiplier.

Proof. Let $T_{2}=T_{1}^{2} T, E=T_{1} T$ and $K=\hat{T}^{-1}(0)$. Then we see that

$$
\hat{T}_{2}(\gamma)= \begin{cases}0 & \text { on } K, \\ (\hat{T}(\gamma))^{-1} & \text { outside } K\end{cases}
$$

and $\hat{E}=\chi_{K^{c}}$, the characteristic function of the complement of $K$. Thus $E$ is an idempotent. Let $T^{\prime}=T+1-E$, where 1 is the identity operator. It is easy to check that $T_{2} T=T_{1}^{2} T^{2}=E^{2}=E, T_{2} E=T_{2}$ and $T E=T$. Therefore

$$
\begin{aligned}
T^{\prime}\left(T_{2}+1-E\right) & =(T+1-E)\left(T_{2}+1-E\right) \\
& =E+T_{2}-T_{2}+T-T+1-E=1 .
\end{aligned}
$$

Hence $T^{\prime}$ is invertible. Finally we note that $T=E T=E(T+1-E)=E T^{\prime}$. Thus $T$ is a product of an idempotent and an invertible multiplier. This completes the proof.

Proof of Theorem 1. The proof of 'if' part is trivial. For the 'only if' part, let $T$ be a multiplier such that $T^{2}(S)$ is closed. Let $K=\hat{T}^{-1}(0)$. Then hull $T^{2}(S)=$ hull $T(S)=K$. Now $T(S)$ is an ideal and $T^{2}(S)=T(T(S))$ is closed. Hence by Lemma $1, \hat{T}$ is bounded away from zero on $K^{c}$, the complement of $K$. Hence $K$ is open and closed and therefore $K$ is a set of 
spectral synthesis. Since $T^{2}(S)$ is closed, we have $T^{2}(S)=k(K)=\{f \in S$ : $\hat{f}=0$ on $K$ \}. But $T^{2}(S) \subset T(S) \subset k(K)$ and therefore $T^{2}(S)=T(S)=$ $k(K)$. Also, if $f \in k(K)$ and $T f=0$ then $f=0$ by uniqueness of Fourier transform. Hence $T^{\prime}$, the restriction of $T$ to $k(K)$ is a continuous bijection of $k(K)$ onto $k(K)$. Therefore, $T^{\prime-1}=T_{0}$ is continuous. Also, for all $f \in k(K)$

$$
\widehat{T_{0} f}(\gamma)= \begin{cases}(\hat{T}(\gamma))^{-1} \hat{f}(\gamma) & \text { on } K^{c}, \\ 0 & \text { on } K .\end{cases}
$$

Consider $T_{1}=T_{0}^{2} \circ T . T_{1}$ is a bounded linear map from $S$ into $S$ such that

$$
\widehat{T_{1} f}(\gamma)= \begin{cases}(\hat{T}(\gamma))^{-1} \hat{f}(\gamma) & \text { on } K^{c}, \\ 0 & \text { on } K .\end{cases}
$$

Thus $T_{1} \in M(S)$ and $T_{1}$ and $T$ satisfy the hypothesis of Lemma 2 . Therefore $T$ is a product of an idempotent and an invertible multiplier and the proof is complete.

3. Proof of Theorem 2. Let $T$ be an isometric multiplier of $S$. Then $T^{2}(S)$ is closed. Thus by Theorem $1, T=E T^{\prime}$, where $E$ is an idempotent and $T^{\prime}$ is an invertible multiplier. Let $K=\hat{E}^{-1}(0)$. Then $K$ is open and closed. If $K$ is nonempty, choose $f \in S$ such that $f \neq 0$ and $\hat{f}$ is supported in $K$. Then $E f=0$ and therefore $T f=0$. But this contradicts the fact that $T$ is an isometry. Thus $K$ is empty and hence $\hat{E}(\gamma)=1$ for all $\gamma \in \Gamma$. Therefore $E=1$ and $T=T^{\prime}$ is invertible and hence surjective.

To prove the rest of the assertion, let $T_{1}$ be the inverse of $T$. Then $T_{1} \in M(S)$ and for all $\gamma \in \Gamma, \hat{T}_{1}(\gamma)=(\hat{T}(\gamma))^{-1}$. Hence for all $\gamma \in \Gamma$, $|\hat{T}(\gamma)| \leqslant\|T\|=1$ and $|\hat{T}(\gamma)|^{-1}=\left|\hat{T}_{1}(\gamma)\right| \leqslant\left\|T_{1}\right\|=1$. Therefore $|\hat{T}(\gamma)|=1$ for all $\gamma \in \Gamma$ and this completes the proof.

Proof of Theorem 3. Let $T$ be a compact multiplier of a Segal algebra $S$ on a noncompact group $G$. Let us take any complex number $\lambda \neq 0$. Then the range of $T-\lambda 1$, where 1 is the identity operator, is closed by Theorem 4.23 of [5]. Since $T-\lambda 1$ is also a multiplier it follows from Lemma 1 that $K=\{\gamma \in \Gamma: \hat{T}(\gamma)=\lambda\}=\operatorname{hull}((T-\lambda \mathbf{1}) S)$ is open and closed. Let $k\left(K^{c}\right)=$ $\{f \in S: \hat{f}(\gamma)=0$ for all $\gamma \notin K\}$. Consider $T^{\prime}$, the restriction of $T$ to $k\left(K^{c}\right)$. Obviously $T^{\prime}=\lambda 1$ on $k\left(K^{c}\right)$. Now, $T^{\prime}$ is compact and therefore $k\left(K^{c}\right)$ is finite dimensional. Let its dimension be $n$. Suppose $K$ is nonempty. Since $G$ is noncompact, $\Gamma$ is nondiscrete. Hence any nonempty open set in $\Gamma$ has infinite number of points. Therefore we can find $(n+1)$ points $x_{1}, x_{2}, \ldots, x_{n+1}$ all belonging to $K$ and compact neighbourhoods $V_{1}, V_{2}, \ldots, V_{n+1}$ of $x_{1}$, $x_{2}, \ldots, x_{n+1}$ respectively such that $V_{i} \cap V_{j}=\varnothing$ for $i \neq j$ and $V_{i} \subset K$ for $i=1,2, \ldots, n+1$. Choose $f_{i} \in S$ for $i=1,2, \ldots, n+1$ such that $\hat{f}_{i}\left(x_{i}\right)=$ 1 and support of $\hat{f}_{i} \subset V_{i}$. Obviously $\left\{f_{i}\right\}_{i=1}^{n+1}$ forms a linearly independent set of $k\left(K^{c}\right)$. But this contradicts the fact that the dimension of $k\left(K^{c}\right)$ is $n$. Hence $K$ is empty and therefore $\hat{T}(\gamma) \neq \lambda$ for all $\gamma \in \Gamma$. Since $\lambda$ is an arbitrary nonzero complex number we conclude that $\hat{T}(\gamma)=0$ for all $\gamma \in \Gamma$. 
Hence $T=0$ and this completes the proof.

4. We note that the arguments of $\$ 2$ apply to any regular, commutative, semisimple, tauberian Banach algebra $A$ satisfying the condition that for each neighbourhood $V$ of any element $\gamma$ of the maximal ideal space $\Gamma$ of $A$ there is a multiplier $T$ of $A$ with Gelfand transform $\hat{T}$ supported by $V, \hat{T}(\gamma)=1$ and $\|T\|<K$, for a fixed constant $K$. This condition is less restrictive than that mentioned in $\$ 4$ of [1] and hence as has been noted already in $\$ 1$, our results are stronger. For any such Banach algebra Theorem 2 will also hold. As has been proved in [3], Theorem 2 is actually true for any regular, commutative, semisimple, tauberian Banach algebra. Theorem 3 can likewise be generalised to any such Banach algebra satisfying the above condition and whose maximal ideal space $\Gamma$ has no isolated points.

\section{REFERENCES}

1. I. Glicksberg, When is $\mu * L_{1}$ closed?, Trans. Amer. Math. Soc. 160 (1971), 419-425.

2. R. Larsen, An introduction to the theory of multipliers, Springer-Verlag, Berlin and New York, 1971.

3. K. Parthasarathy, Segal algebras-some explorations, Doctoral Dissertation, Indian Institute of Technology, Kanpur, 1977.

4. H. Reiter, $L^{1}$-algebra and Segal algebras, Lecture Notes in Math., vol. 231, Springer-Verlag, Berlin and New York, 1971.

5. W. Rudin, Functional analysis, McGraw-Hill, New York, 1973.

6. _ Fourier analysis on groups, Interscience Tracts in Pure and Appl. Math., no. 12, Interscience, New York, 1962.

Department of Mathematics, Indian Institute of Technology, KanPUR-208016, Indi 